\author{
MANVEEN SINGH \\ Jindal Global Law School, O.P. Jindal Global University, India

\section{TRACING THE EVOLUTION OF STANDARDS AND STANDARDS-SETTING ORGANIZATIONS IN THE ICT ERA}

\begin{abstract}
:
Standards and standards-setting organizations (SSOs) have played a crucial role in shaping the innovation landscape for over three decades, especially in the information and communication technologies (ICT) sector. The advancement in mobile telecommunication and the Internet has led to a fundamental change in the way individuals communicate with each other. Devices such as smartphones, tablets, laptops and smart watches bear complex mechanical and technological features and perform multiple functionalities by connecting seamlessly. However, in order for the interoperability of these devices and their functionalities to come through, there is a requirement of a common set of specifications and interfaces, in the form of standards. Standards are widely acknowledged to be the mainstay of modern economy and can lead to an increase in the value of consumer products, as well as increased rates of innovation. The setting of standards and commercializing of innovation at large is facilitated by voluntary associations called SSOs. Competing firms come together under the auspices of SSOs to collaboratively select and adopt uniform technical standards. It is worth noting that the benefits brought about by these standards have a greater visibility in the ICT sector, primarily on account of two reasons. First, in order to make complex technologies work, there is a requirement of hundreds of thousands of patents. Second, there is a strong need for devices and networks to interoperate in the ICT sector, which makes it absolutely necessary to develop common technical standards.
\end{abstract}

SSOs are further tasked with the responsibility of fostering a regime of rapid technological innovation by balancing the interests of their members; their membership comprising of patent owners or standard essential patent (SEP) holders on one hand and implementers or licensees on the other. While the patent owners are involved in research and development $(R \& D)$ and look to maximize their earnings from licensing out their SEPs, the implementers look to seek licenses from SEP holders on terms that are fair, reasonable and non-discriminatory (FRAND), in order to use the patented technology in the manufacturing of standard-compliant end-use products. There is yet, a third category of member companies that are vertically integrated and besides owning SEPs, also operate actively in the downstream market. As members of SSOs, these firms compete in the market on both, horizontal and vertical levels, which gives rise to a possible likelihood of collusion albeit theoretically. It is because of this aspect of standard-setting, that the role of SSOs becomes extremely important.

A pertinent question that arises then is, what are SSOs and how do they function? Furthermore, what is the legality of SSOs and how have they helped in the evolution of industry standards? In an attempt to answer the aforementioned questions, the focus of this paper shall center around standardization and standard-setting organizations, while tracing the evolution of standards and standard-setting activities in the ICT sector. 


\section{Keywords:}

Standards, standardization, SSO, patents, SEP, technology

JEL Classification: 030 